\title{
Magnetic interactions and reversal mechanisms in Co nanowire and nanotube arrays
}

\author{
M. P. Proenca, ${ }^{1,2}$ C. T. Sousa, ${ }^{1}$ J. Escrig, ${ }^{3}$ J. Ventura, ${ }^{1}$ M. Vazquez, ${ }^{2}$ and J. P. Araujo ${ }^{1, a)}$ \\ ${ }^{1}$ IFIMUP and IN - Institute of Nanoscience and Nanotechnology and Dep. Física e Astronomia, \\ Univ. Porto, Rua do Campo Alegre 687, 4169-007 Porto, Portugal \\ ${ }^{2}$ Instituto de Ciencia de Materiales de Madrid, CSIC, 28049 Madrid, Spain \\ ${ }^{3}$ Departamento de Física, Universidad de Santiago de Chile (USACH) and Center for the Development \\ of Nanoscience and Nanotechnology (CEDENNA), Avda. Ecuador 3493, Santiago, Chile
}

(Received 3 December 2012; accepted 20 February 2013; published online 6 March 2013)

\begin{abstract}
Ordered hexagonal arrays of Co nanowires (NWs) and nanotubes (NTs), with diameters between 40 and $65 \mathrm{~nm}$, were prepared by potentiostatic electrodeposition into suitably modified nanoporous alumina templates. The geometrical parameters of the NW/NT arrays were tuned by the pore etching process and deposition conditions. The magnetic interactions between NWs/NTs with different diameters were studied using first-order reversal curves (FORCs). From a quantitative analysis of the FORC measurements, we are able to obtain the profiles of the magnetic interactions and the coercive field distributions. In both NW and NT arrays, the magnetic interactions were found to increase with the diameter of the NWs/NTs, exhibiting higher values for NW arrays. A comparative study of the magnetization reversal processes was also performed by analyzing the angular dependence of the coercivity and correlating the experimental data with theoretical calculations based on a simple analytical model. The magnetization in the NW arrays is found to reverse by the nucleation and propagation of a transverse-like domain wall; on the other hand, for the NT arrays a non-monotonic behavior occurs above a diameter of $\sim 50 \mathrm{~nm}$, revealing a transition between the vortex and transverse reversal modes. (C) 2013 American Institute of Physics. [http://dx.doi.org/10.1063/1.4794335]
\end{abstract}

\section{INTRODUCTION}

With the growing need for the miniaturization of sensors, storage devices and biomedical chips, magnetic nanomaterials have gained much importance in the last years. ${ }^{1-3}$ Nanowires (NWs) and nanotubes (NTs) have thus received increased attention, as they exhibit anisotropic (shape-dependent) collective properties, such as photoluminescence, conductivity, and magnetization. ${ }^{4}$ Most applications use arrays of nanostructures, adding new degrees of freedom as the importance of inter-element coupling increases. The ability to control the NW/NT dimensions (length, inner/outer diameters, and wall thickness), the array configuration and interwire distance, allows the tuning of different energies (magnetostatic, magnetocrystalline, and exchange) to obtain novel phenomena induced by nanoscopic confinement or proximity effects. $^{5-9}$

A thorough understanding of the magnetic properties of NW and NT arrays with tailored geometries is of extreme importance for their implementation in future applications. In particular, new insights on the magnetostatic interactions between nanoelements will highly influence the design of improved devices using magnetic arrays. Interwire interactions are known to affect the magnetic properties of NW and NT arrays, particularly magnetization switching processes. The ability to quantitatively study these interactions can provide an accurate understanding of magnetic nanoscopic effects. A straightforward way of studying the magnetic

${ }^{\text {a)} E l e c t r o n i c ~ a d d r e s s: ~ j e a r a u j o @ f c . u p . p t . ~}$ properties of a system is the analysis of major hysteresis cycles. However, such information is not sufficient for a complete understanding of magnetic interactions. Using the same experimental set-up, but measuring multiple minor hysteresis loops, the so-called first-order reversal curves (FORCs), one can extract quantitative information on the magnetic interactions of nanoelement arrays. ${ }^{10-12}$

Additionally, the ability to tune the magnetization reversal modes of nanoelement arrays by external parameters, such as the applied magnetic field, has also become attractive as it improves the implementation and control of novel devices. Depending on the geometrical specifications of the system under study, three main modes of magnetization reversal have been previously identified: coherent mode (C), where all magnetic moments rotate homogeneously; transverse mode (T), where spins rotate progressively by the nucleation and propagation of a transverse-like domain wall (DW); and vortex mode (V), where a vortex-like DW nucleates and propagates. ${ }^{13}$ In recent reports a simple analytical model has been proposed to understand the magnetization reversal processes by predicting the variation of the coercivity with the angle of applied external magnetic field $(H)$ and allowing the understanding of the magnetization reversal processes for NW and NT arrays with different geometrical parameters. ${ }^{13-15}$

Nanoporous alumina templates (NpATs) have been extensively used for the growth of NWs and NTs as they offer the possibility to build ordered arrays of aligned nanostructures and the ability to easily control the required dimensions by changing the anodization conditions. ${ }^{16,17}$ Controlling the applied potential and the electrolyte type, 
concentration and temperature, one can easily obtain ordered hexagonal arrays of nanopores with diameters between 20 and $200 \mathrm{~nm}$ and interpore distances in the range of $60-500 \mathrm{~nm}^{18}$ The thickness of the NpATs can be tuned from a few nanometers to hundreds of micrometers by varying the anodization time.

Several methods can then be used for the growth of NWs and NTs inside the nanopores of the templates, including sol-gel filling, atomic layer, and electrochemical depositions. ${ }^{19-21}$ For the fabrication of large arrays of metallic NWs and NTs, potentiostatic electrodeposition in NpATs has proved to be a low cost and high yield technique. ${ }^{22}$ Varying the deposition conditions (time, applied potential and electrolyte temperature, $\mathrm{pH}$, and concentration), we can also tune the crystallographic structure and metal alloy composition, changing the respective physical response. ${ }^{23,24}$ Additionally, the accurate coating of the metallic contact (cathode) at the bottom of the pores, where the deposition of the nanostructure initiates, allows the controlled formation of NWs or NTs. ${ }^{25}$

In this work, ordered hexagonal arrays of Co NWs and NTs, with diameters between 40 and $65 \mathrm{~nm}$, were fabricated by template assisted electrodeposition using NpATs. FORC measurements were performed with $H$ along the wire axis, allowing us to obtain quantitative results on the magnetic interwire interactions in NW and NT arrays. These were found stronger in the NW case, increasing with the diameter for both NW and NT arrays. Magnetization reversal modes were also studied for both NW and NT arrays measuring the angular dependence of the coercivity and comparing the experimental data with theoretical analytical calculations. We found that the magnetization in NW arrays reverses by the nucleation and propagation of a transverse DW. In the case of NT arrays, we find a critical diameter of $\sim 50 \mathrm{~nm}$ above which magnetization reverses by a vortex DW.

\section{EXPERIMENTAL DETAILS}

NpATs were prepared by a two-step anodization process of high-purity $\mathrm{Al}$ (99.999\%) disks. ${ }^{16}$ Prior to anodization, $\mathrm{Al}$ disks were cleaned in acetone and ethanol, and electropolished in a solution of $75 \%$ ethanol and $25 \%$ perchloric acid by applying $20 \mathrm{~V}$ for $2 \mathrm{~min}^{26}$ The subsequent anodization processes were performed in $0.3 \mathrm{M}$ oxalic acid at $40 \mathrm{~V}$, maintaining the electrolyte temperature at $\sim 4{ }^{\circ} \mathrm{C}$. To obtain ordered hexagonal nanopore arrays first anodizations were performed for $24 \mathrm{~h}$. The alumina was then removed by chemical etching in an aqueous solution of $0.2 \mathrm{M} \mathrm{H}_{2} \mathrm{CrO}_{4}$ and $0.4 \mathrm{M} \mathrm{H}_{3} \mathrm{PO}_{4}$ at $60{ }^{\circ} \mathrm{C}$, and second anodizations were performed using the same conditions as the first ones, but only for $\sim 20 \mathrm{~h}$, producing membranes with $\sim 50 \mu \mathrm{m}$ in thickness. ${ }^{22}$ The obtained NpATs had pore diameters of $d \sim 35 \pm 4 \mathrm{~nm}$ and interpore distances of $D_{\text {int }} \sim 102 \pm 3 \mathrm{~nm}$.

For the subsequent pore filling of the NpATs using potentiostatic electrodeposition, a circle with $\sim 1 \mathrm{~cm}$ in diameter was opened on the template backside, by chemically etching the $\mathrm{Al}$ substrate in an aqueous solution of $0.2 \mathrm{M} \mathrm{CuCl}_{2}$ and 4.1 $\mathrm{M} \mathrm{HCl}$ at room temperature. The alumina barrier layer present at the bottom of the nanopores was then removed by a controlled chemical etching using $0.5 \mathrm{M} \mathrm{H}_{3} \mathrm{PO}_{4}$. Tuning the time and temperature of this last process allowed us to obtain final nanopore diameters of $d \sim(40,50$, and 65$) \pm 4 \mathrm{~nm}$. For diameters of $d \sim 50$ and $65 \mathrm{~nm}$, the alumina membranes were submerged in $0.5 \mathrm{M} \mathrm{H}_{3} \mathrm{PO}_{4}$ at $19^{\circ} \mathrm{C}$ for 2 and $3 \mathrm{~h}$, respectively. To obtain the smaller pore diameters $(d \sim 40 \mathrm{~nm})$, the solution of $0.5 \mathrm{M} \mathrm{H}_{3} \mathrm{PO}_{4}$ was left in contact only with the bottom of the membranes, at $70^{\circ} \mathrm{C}$, for around $10 \mathrm{~min}$. Finally, an Au metallic contact was sputtered at the opened nanopores side of the NpATs to serve as the working electrode in a three-electrode cell. For the fabrication of NWs, a continuous Au film ( $\sim 120 \mathrm{~nm}$ in thickness) was sputtered at the nanopores' bottom, to completely close its opened end. For the growth of NTs, a thinner metallic contact $(\sim 60 \mathrm{~nm})$ was sputtered, as not to completely close the bottom of the pores, allowing the electrochemical growth of a tubular structure throughout the nanopores. ${ }^{25} \mathrm{~A} \mathrm{Pt}$ mesh was used as the counter electrode and $\mathrm{Ag} / \mathrm{AgCl}$ (in $4 \mathrm{M} \mathrm{KCl}$ ) as the reference electrode ( $0.197 \mathrm{~V}$ vs. standard hydrogen electrode).

The electrodepositions were performed in an aqueous solution of $0.89 \mathrm{M} \mathrm{CoSO}_{4} \cdot 7 \mathrm{H}_{2} \mathrm{O}$ and $0.49 \mathrm{M} \mathrm{H}_{3} \mathrm{BO}_{3}$, at $30{ }^{\circ} \mathrm{C}$, and applying a constant potential of $-1.5 \mathrm{~V}$ vs. $\mathrm{Ag} /$ $\mathrm{AgCl}$, using a Solartron 1480 MultiStat. For the NWs the depositions were performed during $1 \mathrm{~min}$, giving wires' lengths of $L \sim 1.7,3.0$, and $6.5 \mu \mathrm{m}$ for $d \sim 65,50$, and $40 \mathrm{~nm}$, respectively. While for the NTs the deposition times varied from 1 to 4 min, resulting in NTs' lengths of $L \sim 20$, 50 , and $40 \mu \mathrm{m}$ for $d \sim 65,50$, and $40 \mathrm{~nm}$, respectively. Morphological characterizations were performed using a scanning electron microscope (SEM; FEI Quanta 400FEG). Prior to bottom SEM imaging, ion milling was performed to remove the Au contact $(200 \mathrm{~nm})$ and smoothen the NpAT surface. The milling process was carried out using an ion beam sputter deposition system by Commonwealth Scientific Corporation. ${ }^{21}$ The angular dependent magnetic hysteresis loops and FORC measurements were performed in a vibrating sample magnetometer (VSM; LOT-Oriel EV7) at room temperature. For all the magnetic measurements, the samples were saturated under a maximum applied magnetic field of $15 \mathrm{kOe}$.

\section{RESULTS AND DISCUSSION}

Ordered hexagonal arrays of Co NWs and NTs with diameters of $d \sim(40,50$, and 65$) \pm 4 \mathrm{~nm}$, NT wall thicknesses of $t \sim 11 \pm 2 \mathrm{~nm}$, and aspect ratios (length/diameter) higher than 20 were obtained by electrodeposition inside the nanopores. Figure 1 shows bottom (after $200 \mathrm{~nm}$ ion milling) and cross-sectional SEM images of selected samples of Co NW and NT arrays in NpATs, evidencing the high order of the hexagonal array and the pore filling uniformity. In particular, Figs. 1(a) and 1(b) correspond to two ordered NW arrays with different diameters and equal interpore distances, illustrating the control of the interwire spacing by adjusting the NWs diameter. The cross-sectional SEM image presented in Fig. 1(c) evidences the homogeneity of the pore filling and the uniformity of the NWs structure along the pore walls. Finally, Fig. 1(d) shows a SEM image of the bottom of a NpAT filled with Co NTs, after ion milling etching, 

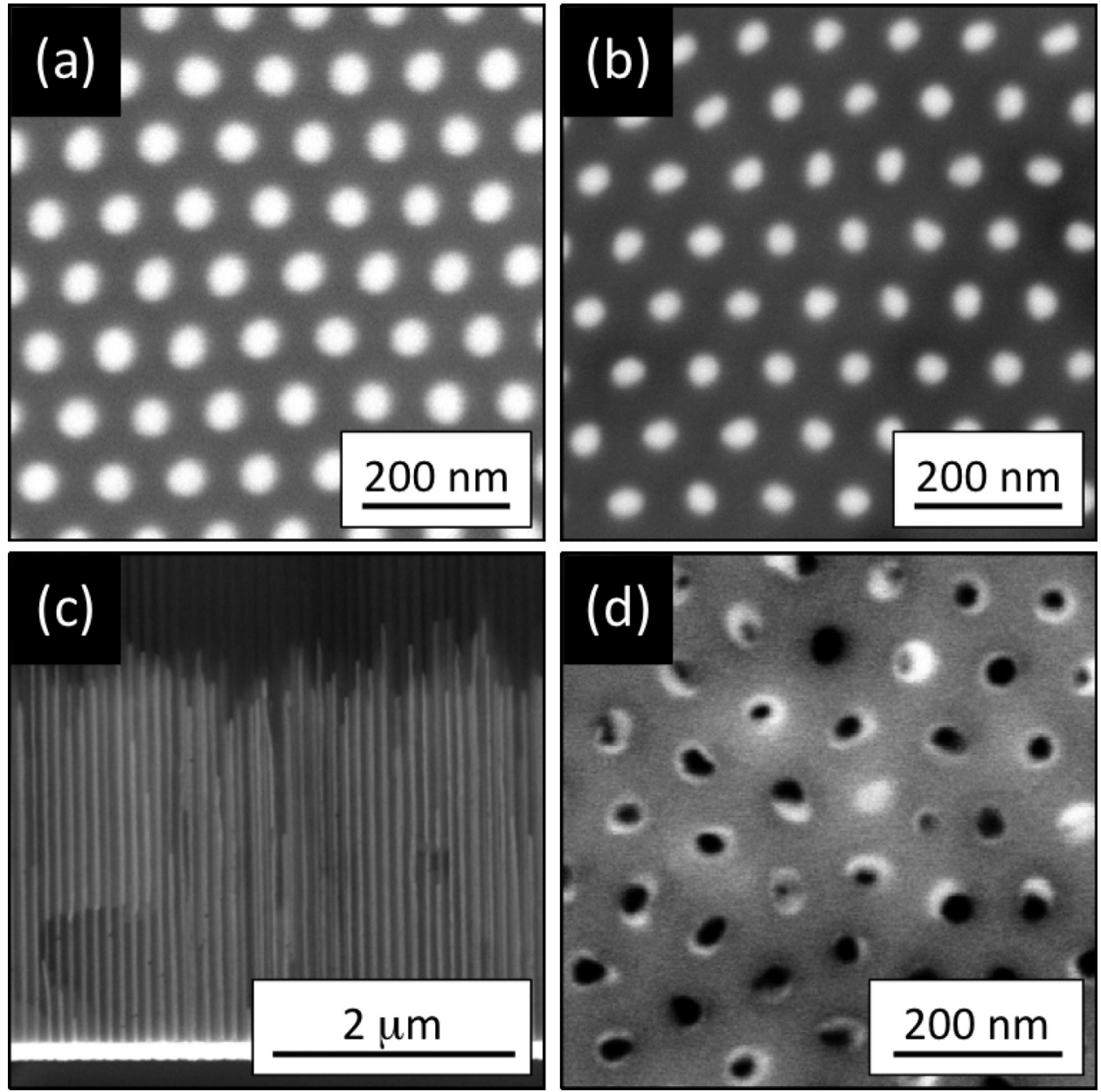

FIG. 1. SEM [(a), (b), and (d)] bottom (after $200 \mathrm{~nm}$ milling) and (c) crosssectional images of Co [(a), (b), and (c)] NW and (d) NT arrays in NpATs with (a) $d \sim 50 \mathrm{~nm}$ and [(b), (c), and (d)] $d \sim 40 \mathrm{~nm}$ and $D_{\text {int }} \sim 102 \mathrm{~nm}$. illustrating the NT shape formed inside the nanopores. Note that, when the Co NTs are removed from the NpAT we observed a fast oxidation process that prevented us from obtaining clear transmission electron microscopy (TEM) images. However, when analyzing the TEM images of similarly fabricated $\mathrm{Ni} \mathrm{NTs}^{27}$ a tubular structure with uniform walls can be found throughout the tube, and thus a similar structure is expected to be also produced when electrodepositing Co NTs.

\section{A. First order reversal curves}

Aiming the accurate understanding of the magnetic interactions in Co NW and NT arrays, we measured FORCs. Although major magnetic hysteresis loops can give information on the coercivity, remanence, and saturation fields of a magnetic system, they will only describe its global (average) behavior. When studying an array of smaller entities (NWs, NTs, nanodots, or nanoparticles) it becomes vital to understand the interactions between the individual particles as they affect the behavior of the array. The FORC method has proved to be an effective way to characterize magnetic interactions and magnetization reversal in nanomagnet arrays. $^{28-31,41}$

To obtain FORCs one first has to saturate the sample applying a large positive magnetic field. Then, we lower $H$ to the so-called reversal field $H_{r}$ (not yet sufficient to saturate the sample in the negative direction), and measure the magnetization $M$ as a function of $H$, varying $H$ from $H_{r}$ to the positive saturation field value. This procedure is then repeated in increasing steps of $H_{r}$, until a complete set of FORCs is obtained (insets of Fig. 2). The FORC distribution is then given by the mixed second derivative of $M\left(H, H_{r}\right)^{10}$

$$
\rho\left(H, H_{r}\right)=-\frac{1}{2} \frac{\partial^{2} M\left(H, H_{r}\right)}{\partial H \partial H_{r}}\left(H>H_{r}\right) .
$$

According to the classical Preisach model, the FORC distribution can be seen as a statistical distribution of square hysteresis curves with coercivity $H_{c}$ and bias field $H_{u}$, called mathematical hysterons. ${ }^{10}$ Various models have been recently developed for the interpretation of FORC results applied to arrays of nanowires. ${ }^{11,28,30,32}$ These models then enable one to extract quantitative information on the coercivity distribution of individual nanowires, interaction field at saturation, and obtain insights into the magnetization reversal mechanism. For a better interpretation of the FORC measurements, it is usual to plot the FORC diagram as a contour plot of the FORC distribution (Fig. 2), with a color scale from minimum (blue) to maximum (red). ${ }^{12}$ It is also convenient to define a new set of coordinates $\left(H_{c}, H_{u}\right)$, that will correspond to the coercive field and interaction field axis, respectively, as follows:

$$
H_{c}=\frac{H-H_{r}}{2}, H_{u}=-\frac{H+H_{r}}{2} .
$$




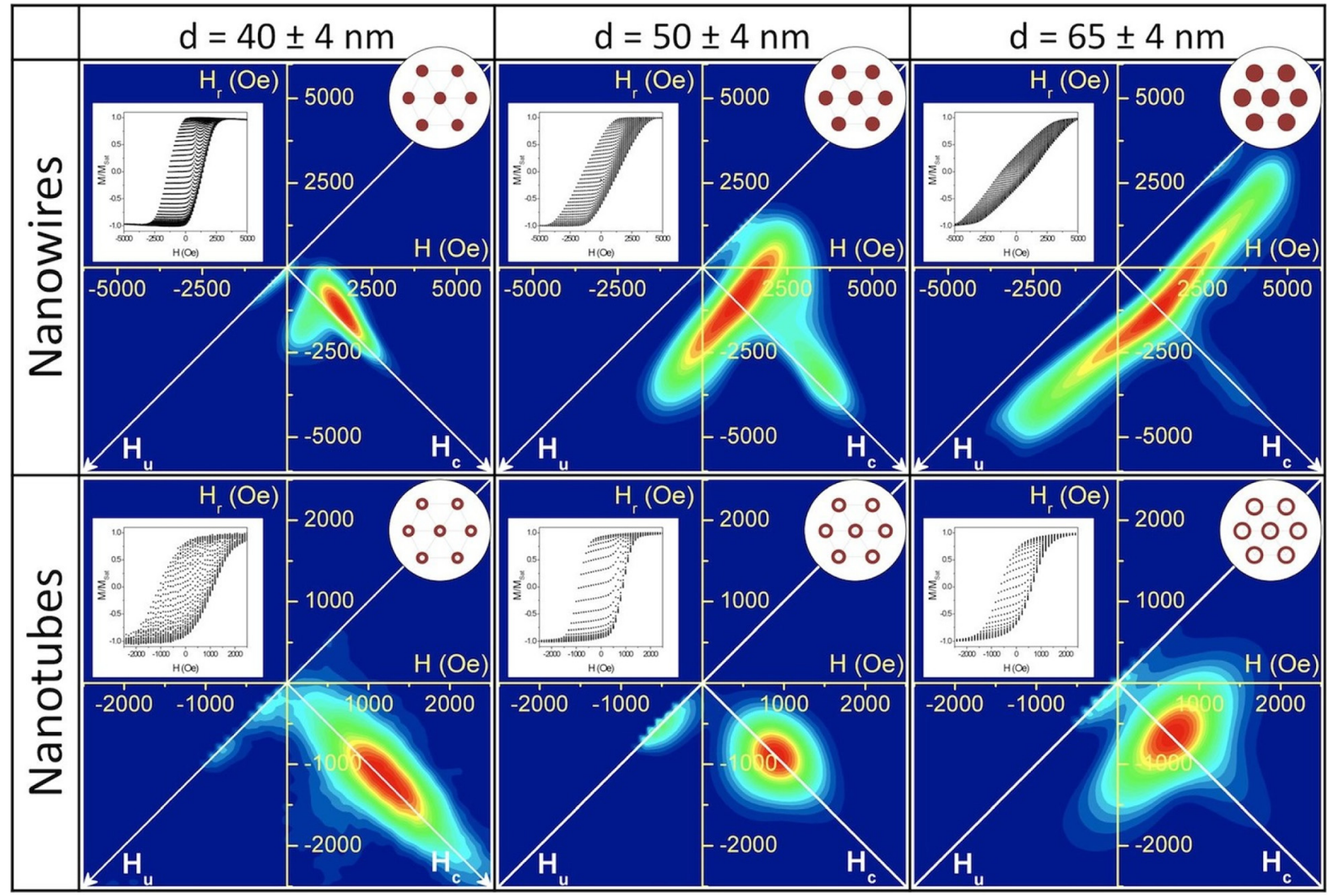

FIG. 2. FORCs (insets) and FORC diagrams of Co NW and NT ordered hexagonal arrays with different diameters and lattice parameter of $\sim 102 \mathrm{~nm}$, when applying the magnetic field parallel to the wire/tube long axis.

Figure 2 shows the FORC results obtained in NW and NT arrays with different diameters and the same center-tocenter wire/tube distances, and $H$ parallel to the long axis. From the overall analysis of the FORC results of all samples, one can observe an increase of the FORC distribution along the $H_{u}$ axis as the diameter increases. This can be ascribed to the increase of magnetostatic interactions among the NWs/ NTs as their diameter increases and the distance between them decreases. The half-width of the FORC distribution along the $H_{u}$ axis $\left(\Delta H_{u}\right)$ can be used as an estimative value of the interaction field between the NWs/NTs at saturation. For most NW array systems, the maximum observed in the FORC diagram can be associated to a good approximation with the average value of the NW coercivity $\left(H_{c}^{F O R C}\right){ }^{28}$ However, for small diameters $(d \sim 40 \mathrm{~nm})$ we found a high coercivity distribution along the $H_{c}$ axis. In these cases the maximum value of the FORC diagram cannot be associated with $H_{c}^{\mathrm{FORC}}$ as previously reported. ${ }^{11,29}$

From the quantitative analysis of the FORC measurements, we calculated $H_{c}^{\mathrm{FORC}}$ and the magnetic interactions $\left(\Delta H_{u}\right)$ between the NWs/NTs. Keeping the distance between the NWs/NTs centers constant, we see that $\Delta H_{u}$ increases with increasing diameter, due to the increased proximity of the neighboring NWs/NTs walls (Fig. 3). ${ }^{30}$ Additionally, the magnetic interactions are found much stronger between NWs than between NTs. However, lowering the NW/NT diameter down to $\sim 35 \mathrm{~nm}$, while keeping the interwire distance, should already lead to a similar behavior for both NW and NT arrays (Fig. 3).
Using the moving Preisach model, ${ }^{28,33}$ that also takes into consideration the interactions between the mathematical hysterons, the interaction field at saturation can be approximated by ${ }^{28}$

$$
H_{\text {int }, \text { sat }}=2 \sigma_{\text {int }}-k,
$$

where $\sigma_{i n t}$ is the standard deviation of the interaction field distribution (assumed constant at a given particle site but

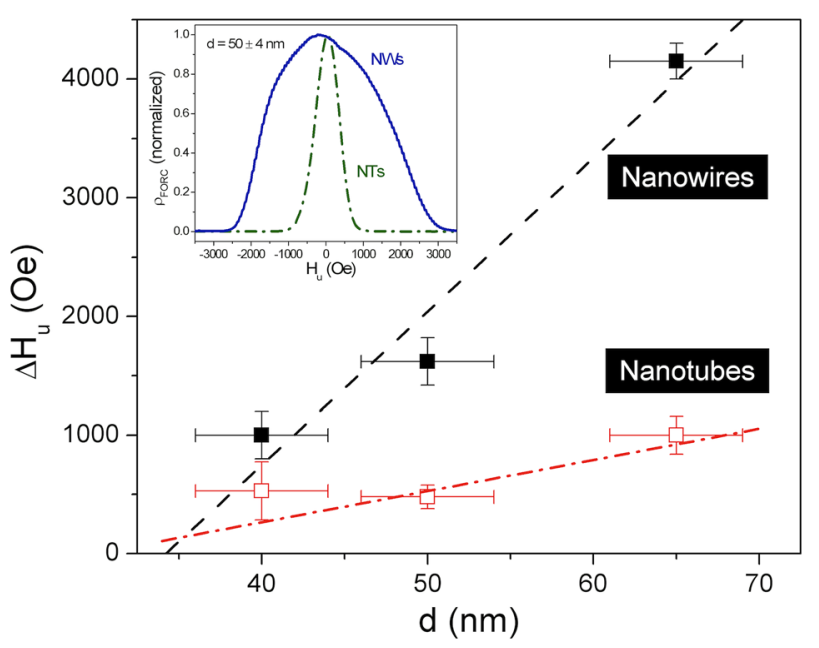

FIG. 3. Magnetic interactions, extracted from the FORC diagrams in Fig. 2, as a function of the wire/tube diameter. Inset shows the cross-section of the FORC diagram along the $H_{u}$ axis for NW and NT arrays with $d \sim 50 \mathrm{~nm}$. 
varying randomly from site to site), ${ }^{34}$ and $k$ is related with the mean interaction field $k M / M_{\text {Sat }}$, that can be parallel $(k>0)$ or antiparallel $(k<0)$ to $M$.

Analyzing, the theoretical FORC diagrams reported by Beron $e t a l .{ }^{28}$ using the moving Preisach model and different combinations of $\left(k, \sigma_{\text {int }}\right)$ values $[(0,250) ;(-500,500)$; $(-250,10)],{ }^{28}$ and comparing them with our experimental data (Table I), we see that our NT arrays behave like systems with $k \sim 0$, while NW arrays are comparable to systems with $|k| \approx\left|\sigma_{\text {int }}\right|$ and $k<0$. The NT arrays can therefore be considered as almost non-interacting systems, where the mean interaction field is close to zero and there is only a small local interaction field (Fig. 2). The NW arrays present a much broader distribution of the interaction field along the $H_{u}$ axis, which is attributed to the presence of a mean interaction field antiparallel to $M(k<0)$. As previously reported, the stray field of the NW array will induce an antiferromagnetic-like coupling between NWs creating a macroscopic demagnetizing field. ${ }^{23,32,35-37}$ The inset of Fig. 3 corresponds to the crosssection along the $H_{u}$ axis for NW and NT arrays with $d \sim 50 \mathrm{~nm}$. A sharp (broad) distribution can be seen for the NT (NW) arrays. From the theoretical FORC diagrams of Ref. 28, sharp peaks were obtained when considering $\Delta H_{u}=H_{\text {int.sat }}$ with $k \sim 0$ and $\sigma_{\text {int }}=250 \mathrm{Oe}$, while broader distributions resulted when using $k=-500 \mathrm{Oe}$ and $\sigma_{\text {int }}=500$ Oe. In fact, for the NW and NT arrays with $d \sim 50 \mathrm{~nm}$, we estimated $\Delta H_{u} \approx 1500$ and $500 \mathrm{Oe}$, respectively. If we consider $|k|=\left|\sigma_{\text {int }}\right|$ for the NW array and $k=0$ for the NT array, we obtain $|k|=\left|\sigma_{\text {int }}\right|=500 \mathrm{Oe}$ and $\sigma_{\text {int }}=250 \mathrm{Oe}$, respectively. The good agreement found between the experimental cross-sectional plots along the $H_{u}$ axis (inset of Fig. 3) and the theoretical FORC diagrams reported in Ref. 28, reinforces our statement that the mean interaction field for NT arrays is very low, while that of NW arrays is much stronger and antiparallel to $M$. It has also been reported from theoretical calculations, ${ }^{28}$ that the distribution top along the $H_{u}$ axis becomes flatter with increasing $|k| / \sigma_{\text {int }}$, indicating an array where all nanomagnets experience similar magnetostatic interactions. In the inset of Fig. 3, we can already observe a flatter top in the $H_{u}$ profile of the NW arrays, revealing a more homogeneous distribution of magnetostatic interactions between NWs than between NTs.

Figure 4 shows a comparison between $H_{c}^{F O R C}$ and the coercive field measured from the major hysteresis loops $\left(H_{c}^{H y s t}\right)$, with the applied field parallel to the wire axis, for both NW and NT arrays. The decrease of $H_{c}^{H y s t}$ and $H_{c}^{F O R C}$ with the increase of NW/NT diameter is confirmed. For the NT arrays, we found $H_{c}^{F O R C} \approx H_{c}^{H y s t}$. Since $H_{c}^{F O R C}$ is assumed to be

TABLE I. Interaction field values $\left(\Delta H_{u}\right)$ extracted from the FORC diagrams and respective estimated $\left(\sigma_{\text {int }}, k\right)$ values for Ni NW and NT arrays with different diameters.

\begin{tabular}{lccccc}
\hline \hline & \multicolumn{2}{c}{ NTs $(k \approx 0$ Oe $)$} & & \multicolumn{2}{c}{ NWs } \\
\cline { 2 - 3 } \cline { 5 - 6 }$d(\mathrm{~nm})$ & $\Delta H_{u}(\mathrm{Oe})$ & $\sigma_{\text {int }}(\mathrm{Oe})$ & & $\Delta H_{u}(\mathrm{Oe})$ & $|k| \approx\left|\sigma_{\text {int }}\right|(\mathrm{Oe})$ \\
\hline 40 & 500 & 250 & & 1050 & 350 \\
50 & 500 & 250 & & 1500 & 500 \\
65 & 900 & 450 & & 4050 & 1350 \\
\hline
\end{tabular}

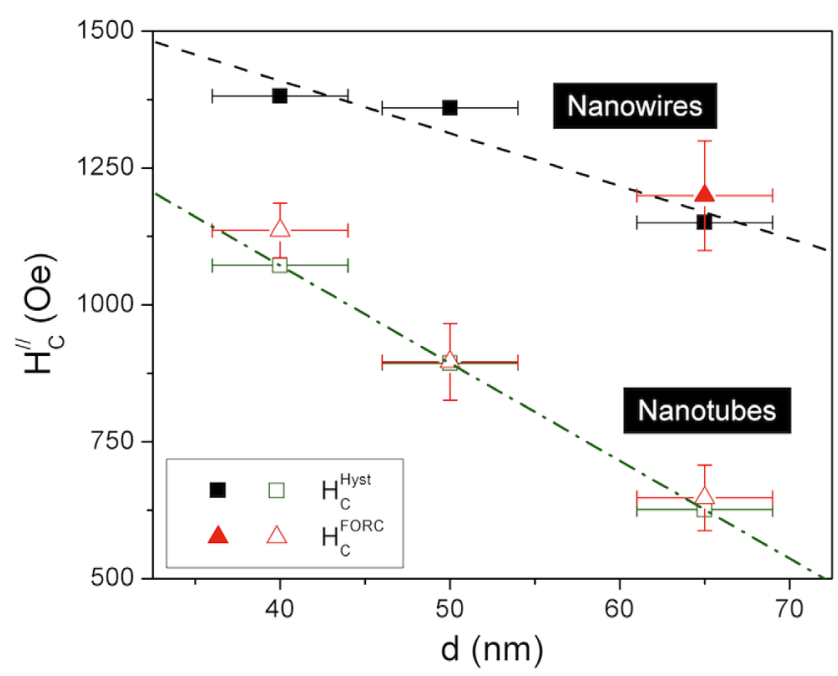

FIG. 4. Coercive field along the parallel direction, extracted from the maximum value of the FORC diagrams $\left(H_{c}^{\mathrm{FORC}}\right)$ in Fig. 2, and from the major magnetic hysteresis loops $\left(H_{c}^{H y s t}\right)$, as a function of the NWs/NTs diameter.

related with the switching field of single nanoelements, the fact that its value is similar to $H_{c}^{H y s t}$ is an additional evidence that the mean interaction field can be neglected in NT array systems. For the NW arrays, due to the high coercivity distribution present in the FORC diagrams for $d \sim 40$ and $50 \mathrm{~nm}$, we were not able to extract the correct value of $H_{c}^{F O R C}$. One should also note that the magnetization reversal mechanisms reported for NWs are much more complicated than the ones expected in NTs, which may explain the sharper and betterdefined distribution of coercivities exhibited in the latter case (Fig. 2). Additionally, the FORC diagrams presented in Fig. 2 illustrate a coercivity distribution along the $H_{c}$ axis for all NW arrays, and for NT arrays with smaller outer diameters $(\sim 40 \mathrm{~nm})$. However, for NT arrays with $d \sim 50$ and $65 \mathrm{~nm}$ such coercivity distribution is practically inexistent. This may be attributed to different magnetization reversal mechanisms for the NW and NT arrays, specially at larger diameters $(\sim 50-65 \mathrm{~nm})$, where the differences in $H_{c}^{F O R C}$ distributions are more pronounced. The FORC analysis thus allows one to extract quantitative knowledge on the interaction field, average individual coercivity and overall array anisotropy, which can help in the interpretation of the magnetization reversal mechanisms. However, for a deeper understanding of the reversal processes occurring in NW or NT arrays, additional information must be retrieved using complementary measurements, such as the angular dependence of the coercivity that will be presented in Sec. III B.

\section{B. Magnetization reversal}

In order to obtain further insights into the magnetic properties of Co NW and NT arrays, magnetic hysteresis loops were measured at different angles $(\theta)$ between $H$ and the NW/NT long axis. Figure 5 shows the magnetic hysteresis loops measured for Co NW and NT arrays, with $d \sim 50 \mathrm{~nm}$. A monotonic decrease of the coercive field $\left(H_{c}\right)$, from $\theta=0^{\circ}$ to $90^{\circ}$ can be seen for the NW arrays, as expected. However, for NT arrays, a non-monotonic behavior of $H_{c}$ is present, evidenced by an increase in $H_{c}$ for $\theta>0^{\circ}$, followed by a decreasing trend for $\theta>60^{\circ}$. 


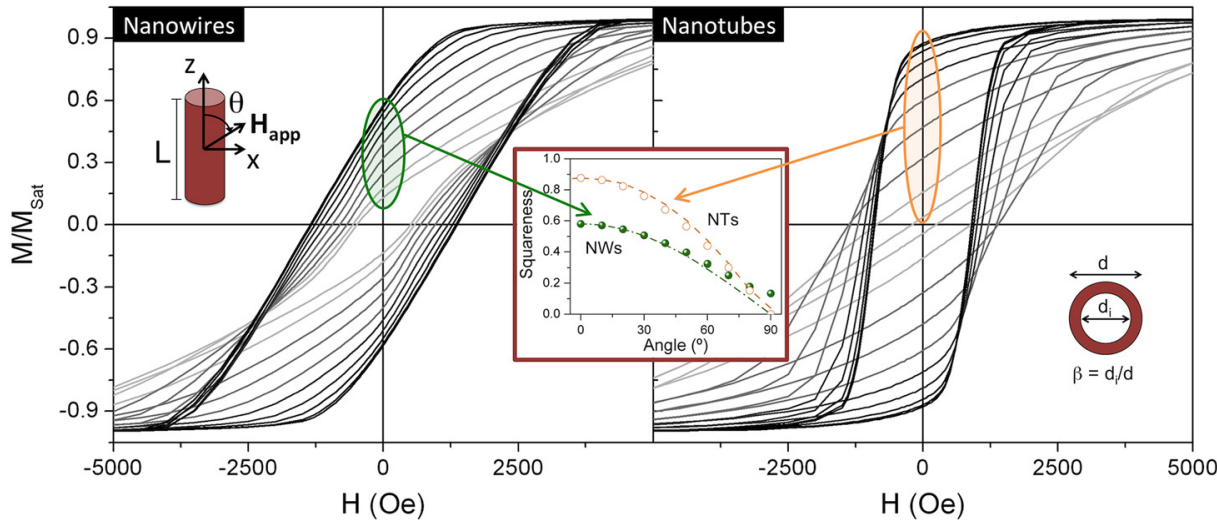

FIG. 5. Magnetic hysteresis loops of ordered Co NW (left) and NT (right) arrays with $d \sim 50 \mathrm{~nm}$ and $d_{i} \sim 30 \mathrm{~nm}$, measured at different angles of $H$, in $10^{\circ}$ steps. Inset shows the angular dependence of squareness (dots-experimental data and line-theoretical calculations).
The squareness $(S)$, defined as the ratio between remanence $\left(M_{R}\right)$ and saturation $\left(M_{S a t}\right)$ magnetization, was measured for all samples as a function of $\theta$ (inset of Fig. 5). A maximum $S$ value was observed when $\theta=0^{\circ}$, corresponding to $H$ parallel to the NW/NT axis. Inset of Fig. 5 shows a good agreement between the experimental results (dots) and theoretical calculations (line), performed using $S(\theta)=S_{0}|\cos \theta|$, where $S_{0}=S\left(\theta=0^{\circ}\right){ }^{15}$

To investigate the magnetization reversal processes of Co NW and NT arrays, we analyzed the angular dependence of $H_{c}$. Previous works reported analytical calculations of the magnetic reversal modes [coherent $(\mathrm{C})$ magnetization rotation, and transverse $(\mathrm{T})$ and vortex-like $(\mathrm{V})$ domain walls] that are energetically more favorable for high aspect ratio NWs and NTs. ${ }^{8,13-15,20,27,38-40}$ For analytical calculations it is important to define the parameter $\beta=d_{i} / d$, where $d_{i}$ and $d$ are the NT inner and outer diameters, respectively, (Fig. 5).

Figure 6 shows the angular dependence of the coercivity for Co NW and NT arrays. From the analytical calculations one expects the magnetization reversal mechanism to correspond to the mode that offers the lowest coercivity values. From the good agreement obtained between our experimental results and the analytical calculations of the angular dependence of coercivity (Fig. 6), we can identify the reversal processes that occur in each NW/NT array system. One should note that the model used for the analytical calculations does not take into consideration the magnetic interactions between the NWs/NTs. However, the FORC analysis presented in Sec. III A allowed us to deduce that the NT arrays behave as almost non-interacting systems, while the NWs exhibit higher values of magnetostatic interactions. This can explain the better agreement found between the analytical calculations and the experimental data for the NT arrays (Fig. 6).

For all the Co NW arrays studied $(d \sim 40-65 \mathrm{~nm})$ we can deduce that the spins reverse by the nucleation and propagation of a transverse DW [Figs. 6(a)-6(c)]. As previously reported, ${ }^{13}$ the magnetization on a NW with a length larger than the domain wall width is expected to reverse by the nucleation and propagation of a DW.

For Co NT arrays with small diameters $(\$ 40 \mathrm{~nm})$ the reversal mode was also found to occur by the nucleation and propagation of a transverse DW [Fig. 6(d)]. However, when the NTs outer diameter becomes larger than $\sim 50 \mathrm{~nm}$, a transition between two different reversal modes can be identified [Figs. 6(e) and 6(f)]. At small angles of $H$ the nucleation of a vortex wall was found to be energetically favorable. Only for higher angles, where $H$ is almost perpendicular to the NT long axis, is the reversal mechanism given by the propagation of a transverse DW. Comparing NT arrays with $d \sim 50$ and $65 \mathrm{~nm}$ [Figs. 6(e) and 6(f), respectively], we can also observe that the critical angle $\left(\theta_{c}\right)$ of $H$ at which the transition from a vortex to a transverse DW occurs depends on the NT outer diameter. For $d \sim 50 \mathrm{~nm}$, we obtained $\theta_{c} \sim 55^{\circ}$, while for $d \sim 65 \mathrm{~nm}$, the critical transition angle reaches $\theta_{c} \sim 67^{\circ}$. One should note that the outer and inner diameters of a NT array will highly influence the type of DW that is energetically more favorable to nucleate. Therefore, tuning the

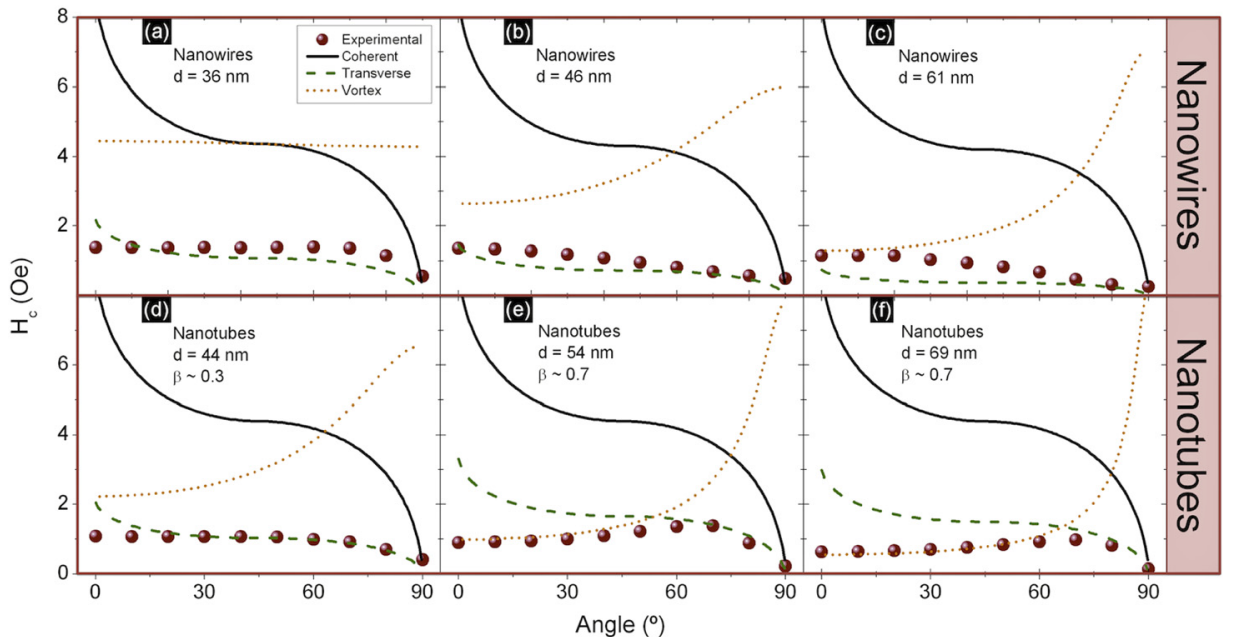

FIG. 6. Angular dependence of the coercive field measured experimentally (dots) and calculated analytically (C mode: black solid; T mode: green dashed; and V mode: orange dotted) for Co NW and NT arrays in NpATs with different outer diameters. 
outer/inner diameters of the NT arrays allows one to control the magnetization reversal mechanisms for the different angles of applied magnetic field.

\section{CONCLUSIONS}

The here presented results allowed us to deduce the magnetization reversal mechanisms in both studied systems. While Co NW arrays with $d \sim 40-65 \mathrm{~nm}$ and NT arrays with $d \sim 40 \mathrm{~nm}$ reverse their magnetization by the nucleation and propagation of a transverse-like DW, NT arrays with $d \sim$ $50-65 \mathrm{~nm}$ nucleate a vortex-like DW. On the other hand, the FORC diagrams shown in Fig. 2 reveal a coercivity distribution along the $H_{c}$ axis in all NW arrays and NT arrays with $d \sim 40 \mathrm{~nm}$. However, for the remaining NT arrays such coercivity distribution is practically inexistent. This shows that magnetization reversal by the nucleation and propagation of a transverse wall increases the coercivity distribution along the $H_{c}$ axis of the FORC diagram, while the nucleation and propagation of a vortex DW does not affect the corresponding coercivity distribution.

Also, the quantitative analysis of FORC measurements allowed us to obtain the profiles of magnetic interaction and coercive field distributions. NW arrays exhibited stronger magnetic interactions than the NT arrays, although in both cases they were found to increase with increasing diameter of the NWs/NTs. The angular dependence of the coercivity of Co NW and NT arrays with different diameters (between 40 and $65 \mathrm{~nm}$ ) was also measured. For NTs with $d \geq 50 \mathrm{~nm}$, we reported experimental evidence for an angular dependent transition of the magnetization reversal modes, which had been theoretically predicted. Analytical calculations allowed us to identify a transverse reversal mode for NW arrays with $d \leq 70 \mathrm{~nm}$ and NT arrays with $d<50 \mathrm{~nm}$, and a transition between vortex and transverse reversal modes for NTs with $d \geq 50 \mathrm{~nm}$.

\section{ACKNOWLEDGMENTS}

M.P.P. and C.T.S. are thankful to FCT for doctoral and post-doctoral Grants SFRH/BD/43440/2008 and SFRH/BPD/ 82010/2011, respectively. J.E. acknowledges financial support from FONDECYT 1110784, Grant ICM P10-061-F by the Fondo de Innovación para la Competitividad-MINECON, AFOSR FA9550-11-1-0347 and the Financiamiento Basal para Centros Científicos y Tecnológicos de Excelencia FB0807. J.V. acknowledges financial support through FSE/ POPH. M.V. thanks the Spanish Ministry of Economia $y$ Competitividad, MEC, under project MAT2010-20798-C0501 and Spanish-Chilean CSIC-USACH Bilateral Project 2010CL0018. J.P.A. also thanks the Fundação Gulbenkian for its financial support within the "Programa Gulbenkian de Estímulo à Investigação Científica." The authors acknowledge funding from FCT through the Associated Laboratory-IN.

${ }^{1}$ P. P. Freitas, F. A. Cardoso, V. C. Martins, S. A. M. Martins, J. Loureiro, J. Amaral, R. C. Chaves, S. Cardoso, L. P. Fonseca, A. M. Sebastiao et al., Lab Chip 12, 546 (2012).

${ }^{2}$ G. Polizos, V. Tomer, E. Manias, and C. A. Randall, J. Appl. Phys. 108, 074117 (2010).
${ }^{3}$ T. Kawahara, K. Ito, R. Takemura, and H. Ohno, Microelectron. Reliab. 52, 613 (2012).

${ }^{4}$ K. D. Sattler, Handbook of Nanophysics: Nanotubes and Nanowires (CRC Press, USA, 2010).

${ }^{5}$ C. A. Ross, Annu. Rev. Mater. Res. 31, 203 (2001).

${ }^{6}$ J. M. D. Coey, Magnetism and Magnetic Materials (Cambridge University Press, New York, 2009).

${ }^{7}$ S. M. Reddy, J. J. Park, S.-M. Na, M. M. Maqableh, A. B. Flatau, and B. J. H. Stadler, Adv. Funct. Mater. 21, 4677 (2011).

${ }^{8}$ M. Yan, C. Andreas, A. Kakay, F. Garcia-Sanchez, and R. Hertel, Appl. Phys. Lett. 99, 122505 (2011).

${ }^{9}$ D. Serantes, V. Vega, W. O. Rosa, V. M. Prida, B. Hernando, M. Pereiro, and D. Baldomir, Phys. Rev. B 86, 104431 (2012).

${ }^{10}$ I. D. Mayergoyz, J. Appl. Phys. 57, 3803 (1985).

${ }^{11}$ F. Beron, Ph.D. thesis, Ecole Polytechnique de Montreal, 2008.

${ }^{12}$ F. Beron, L.-P. Carignan, D. Menard, and A. Yelon, in Electrodeposited Nanowires and Their Applications, edited by N. Lupu (INTECH, Croatia, 2010).

${ }^{13}$ P. Landeros, S. Allende, J. Escrig, E. Salcedo, D. Altbir, and E. E. Vogel, Appl. Phys. Lett. 90, 102501 (2007).

${ }^{14}$ S. Allende, J. Escrig, D. Altbir, E. Salcedo, and M. Bahiana, Eur. Phys. J. B 66, 37 (2008).

${ }^{15}$ R. Lavin, J. C. Denardin, J. Escrig, D. Altbir, A. Cortes, and H. Gomez, J. Appl. Phys. 106, 103903 (2009).

${ }^{16}$ H. Masuda and K. Fukuda, Science 268, 1466 (1995).

${ }^{17}$ K. Nielsch, F. Muller, and A.-P. Li, Adv. Mater. 12, 582 (2000).

${ }^{18}$ M. P. Proenca, C. T. Sousa, D. C. Leitao, J. Ventura, J. B. Sousa, and J. P. Araujo, J. Non-Cryst. Solids 354, 5238 (2008).

${ }^{19}$ C. T. Sousa, A. M. L. Lopes, M. P. Proenca, D. C. Leitao, J. G. Correia, and J. P. Araujo, J. Nanosci. Nanotechnol. 9, 6084 (2009).

${ }^{20}$ J. Escrig, J. Bachmann, J. Jing, M. Daub, D. Altbir, and K. Nielsch, Phys. Rev. B 77, 214421 (2008)

${ }^{21}$ C. T. Sousa, D. C. Leitao, M. P. Proenca, A. Apolinario, J. G. Correia, J. Ventura, and J. P. Araujo, Nanotechnology 22, 315602 (2011).

${ }^{22}$ M. P. Proenca, C. T. Sousa, J. Ventura, M. Vazquez, and J. P. Araujo, Electrochim. Acta 72, 215 (2012).

${ }^{23}$ L. G. Vivas, M. Vazquez, J. Escrig, S. Allende, D. Altbir, D. C. Leitao, and J. P. Araujo, Phys. Rev. B 85, 035439 (2012).

${ }^{24}$ L. G. Vivas, J. Escrig, D. G. Trabada, G. A. Badini-Confalonieri, and M. Vazquez, Appl. Phys. Lett. 100, 252405 (2012).

${ }^{25}$ M. P. Proenca, C. T. Sousa, J. Ventura, M. Vazquez, and J. P. Araujo, Nanoscale Res. Lett. 7, 280 (2012).

${ }^{26}$ D. C. Leitao, A. Apolinario, C. T. Sousa, J. Ventura, J. B. Sousa, M. Vazquez, and J. P. Araujo, J. Phys. Chem. C 115, 8567 (2011).

${ }^{27}$ M. P. Proenca, C. T. Sousa, J. Ventura, J. P. Araujo, J. Escrig, and M. Vazquez, SPIN 2, 1250014 (2012).

${ }^{28}$ F. Beron, L. Clime, M. Ciureanu, D. Menard, R. W. Cochrane, and A. Yelon, J. Nanosci. Nanotechnol. 8, 2944 (2008).

${ }^{29}$ C. Pike, C. Ross, R. Scalettar, and G. Zimanyi, Phys. Rev. B 71, 134407 (2005).

${ }^{30}$ A. Rotaru, J.-H. Lim, D. Lenormand, A. Diaconu, J. B. Wiley, P. Postolache, A. Stancu, and L. Spinu, Phys. Rev. B 84, 134431 (2011).

${ }^{31}$ L. Spinu, A. Stancu, C. Radu, F. Li, and J. B. Wiley, IEEE Trans. Magn. 40, 2116 (2004)

${ }^{32}$ C. Pike, Phys. Rev. B 68, 104424 (2003).

${ }^{33}$ I. D. Mayergoyz, Mathematical Models of Hysteresis and Their Applications (Elsevier, Amsterdam, 2003), Vol. 103.

${ }^{34}$ C. R. Pike, A. P. Roberts, and K. L. Verosub, J. Appl. Phys. 85, 6660 (1999).

${ }^{35}$ K. Nielsch, R. B. Wehrspohn, J. Barthel, J. Kirschner, U. Gosele, S. F. Fischer, and H. Kronmuller, Appl. Phys. Lett. 79, 1360 (2001).

${ }^{36}$ R. Ferré, K. Ounadjela, J. M. George, L. Piraux, and S. Dubois, Phys. Rev. B 56, 14066 (1997).

${ }^{37}$ F. Beron, D. Menard, and A. Yelon, J. Appl. Phys. 103, $07 \mathrm{D} 908$ (2008).

${ }^{38}$ O. Albrecht, R. Zierold, S. Allende, J. Escrig, C. Patzig, B. Rauschenbach, K. Nielsch, and D. Gorlitz, J. Appl. Phys. 109, 093910 (2011).

${ }^{39}$ R. Lavin, J. C. Denardin, A. P. Espejo, A. Cortes, and H. Gomez, J. Appl. Phys. 107, 09B504 (2010).

${ }^{40}$ J. Escrig, M. Daub, P. Landeros, K. Nielsch, and D. Altbir, Nanotechnology 18, 445706 (2007).

${ }^{41}$ M. Almasi Kashi, A. Ramazani, and A. S. Esmaeily, IEEE Trans. Magn. 49, 1167 (2013) 